\title{
Collaborations between Public Health and Computer Science: A Path Worth Pursuing
}

\author{
Jennifer A. Epstein* \\ Department of Public Health, Weill Cornell Medical College, New York, USA \\ *Corresponding author: jepstein@med.cornell.edu
}

Received January 01, 2013; Revised August 01, 2013; Accepted August 02, 2013

\begin{abstract}
Collaboration between public health and computer science shows much promise. The history of the two disciplines is discussed. Examples of recent efforts at collaboration are described to give a flavor of where this path may be going. These studies ranged from some showing effectiveness to others demonstrating the infancy of the collaboration at this point in time. Technology and computer science offer public health many new technological advances by making further use of the internet, enhancements in public health informatics, using computer science to accomplish clinical work gathered by physicians to name a few examples. Still other computer science research concentrations such as animation and virtual reality deserve further exploration. Pursuing this path is certainly worth taking to further advance knowledge and the mission of public health primarily through collaborative efforts taken by individuals/teams in the two disciplines. A collaboration with computer science will help bring public health to meet the $21^{\text {st }}$ century both in terms of challenges and technology.
\end{abstract}

Keywords: public health in the $21^{\text {st }}$ century, public health informatics, health promotion, health outcomes, collaboration with computer science

Cite This Article: Epstein, Jennifer A., "Collaborations Between Public Health and Computer Science: A Path Worth Pursuing." American Journal of Public Health Research 1, no. 7 (2013): 166-170. doi: 10.12691/ajphr-1-7-4.

\section{Introduction}

Collaborations across different fields lead to new and creative techniques. Public health can greatly benefit from interaction with computer science. For example, the latest innovations in computer science, the ever present allure of the internet plus mobile devices and developing computer systems are all useful ways to use the latest developments for the mission of public health. Moreover, these current advances in the electronic age are well suited to continue or pursue such collaborations with computer science. Indeed, some headway has been made on this course as public health and computer science have combined in ways to benefit the public's health, better serve people's health and understand the public's health. Public health is constantly evolving based on the prevalent diseases and the available technology. The American Public Health Association (APHA) defines public health as prevention. More specifically, public health is the practice of preventing disease and promoting good health within groups of people, from small communities to entire countries. Furthermore, the APHA states that public health professionals rely on policy and research strategies. Finally, the APHA explains that public health is not a single discipline but made of professionals from many fields who protect the health of a population, including health educators, public policymakers, scientists and researchers from other disciplines, public health physicians, public health nurses, social workers, epidemiologists, nutritionists, community planners. Computer science is another discipline that can be incorporated into the mission of public health during the $21^{\text {st }}$ century either as professionals joining the field (e.g. public health informatics) or scientists and researchers who collaborate with other public health professionals to solve problems in public health with computers and/or technology. Brief overviews of the history of public health and computer science will be covered. Next, several examples from a few different domains of public health showing how computer science has further moved or begun to move this collaboration forward into the $21^{\text {st }}$ century.

The purpose is to demonstrate the promising advances that have or could be made and encourage continued engagement in this opportunity for collaboration between public health and computer science. E-health and m-health using the internet and mobile devices respectively are among the ways that public health and computer science are expected to collaborate to address public health issues with the latest technology of the $21^{\text {st }}$ century and not remain tied to methods of the past. Specifically, both the internet and mobile devices in the form of e-health and mhealth are developing into ways to inform the public about health issues. E-health and m-health both need to make the best use of these technologies to go beyond simply serving as a text-based or one-way information sources. 


\section{Brief Historical Background of Public Health and Computer Science}

\subsection{Public Health}

Public health in the United States focused on prevailing health problems of the time. Cancer and other chronic conditions predominated in the twentieth century, the way infectious diseases had plagued the nineteenth century; science and technology became major influences in American life, which both helped change public health and medicine [1]. In the twentieth century ten major public health advances have been cited by the CDC [2]. They are vaccination, motor-vehicle safety, safer workplaces, control of infectious diseases, decline in deaths from coronary heart disease and stroke, safe and healthier foods, healthier mothers and babies, family planning, fluoridation of drinking water and recognition of tobacco use as a health hazard.

To elaborate more specifically, vaccination and control of many infectious diseases decreased many deaths in the prior century which occurred in the $19^{\text {th }}$ century (e.g., polio, smallpox, yellow fever). Engineers made vehicles and highways safer and public health efforts to encourage people to use safety belts, car seats for babies/young children and motorcycle helmets decreased motor-vehicle deaths. Safer workplaces have come under better control as deaths related to mining, manufacturing, construction and transportation decreased. Clean water and improved sanitation decreased infections, as did antibiotic treatments. The surgeon general's seminal 1964 report on the dangers of smoking with subsequent tobacco control have helped decrease preventable deaths. Public health advances combined to greatly increase life expectancy for people living in the United States by 25 years [2]. Although these ten public health advances occurred in the United States, other countries have benefitted from the discoveries as other developed countries also made medical and scientific progress translating to the public health front. In addition, more developed nations have tried to help less developed nations through the efforts of the Peace Corps and various health organizations with an international scope.

Some of the examples of public health advances illustrated the combination of public health and technology. Others showed medical breakthroughs implemented by public health officials. Further highlighting the importance of public health, it has been stated that 80 to $90 \%$ of all cancers may be caused by environmental or lifestyle factors rather than genetics [3]. In addition, second hand smoke was killing 3,000 people annually [4] but many states in the USA enacted a variety of laws to protect the rights of non-smokers by creating smoke-free zones at the workplace, in restaurants and other public places. The field of public health has expanded to incorporate multiple disciplines such as biostatistics, epidemiology, health outcomes, health policy, public health informatics, health promotion and prevention, and community health. Various disciplines from public health are in the position to make advances through collaborations with computer science discipline.

\subsubsection{Computer Science}

Computer science is another field that has changed over the years. The field has been described as being born in the 1940s with the confluence of algorithm theory, mathematical logic, and the invention of the storedprogram electronic computer [5]. World War II spawned the electronic computer. At this point, the military allies were using computers to help break the German's computer code. Furthermore, by the early 1960s the first academic departments and degree programs began. During this decade, operating systems for these computers or mainframes were developed. For example, the ARPAnet the precursor to the internet was started and a variety of computer languages were developed. Until the 1980s, the field viewed computers as number crunchers, symbol manipulators and data processors; however, the personal computer and internet broadened the focus to coordination and communication [5]. In the 1990s, computers were getting smaller with laptops becoming prevalent and nanotechnology is currently in development. Now into the $21^{\text {st }}$ century, computer devices are growing even smaller with tablets and other mobile devices with greater functionality including access to the internet, communication and entertainment.

Currently, computer science encompasses a wide variety of areas of interest including algorithms and data structures, programming language theory, concurrent, parallel and distributed systems, databases and information retrieval, machine learning, artificial intelligence, natural language processing, robotics, image processing, Big Data, computer network, computer security and privacy, informatics, computer graphics, sensors, virtual reality among others. Computer science and its various subfields are in a position to interact with other disciplines, including public health.

\section{Examples of the Collaboration between Public Health and Computer Science and its Future}

Cornell University and Technion were chosen to create a team that won New York City's mayor's plan to create a new graduate school of applied sciences. The New York Times reported [6]: “Cornell's engineering and computer science programs rank among the nation's top $10 \ldots$ and it knows New York City, home to its medical school and an array of other programs. Technion...is the one of the world's great high-tech business zones, with alumni running hundreds of companies near its Haifa campus.” Technion has also been referred to as the MIT of Israel. Cornell/Ithaca and Cornell's medical school, including its Department of Public Health, will participate in the research opportunities this union creates. A mere 13 months after being awarded the prize by New York City's Mayor Bloomberg, the new science school opened in a temporary location during January 2013 as NYC Cornell Tech. The creation of this team effort opens opportunities for future ventures. The first class of students enrolled in the one-year masters program in computer science. Eventually rather than traditional departments, "hubs" will exist including connective media, healthier life and built environment. What follows are a few examples of various types of collaborations between public health and 
computer science in recent years broken down by several different areas within public health. Like the venture between Cornell and Technion, information technology is the common thread.

\subsection{Health Promotion}

A meta-analysis of 75 computer-delivered interventions for health promotion from 1988 to 2007 found that participants who received a computer-delivered intervention relative to assessment only controls improved on several health behaviors (nutrition, tobacco use, substance use, safer sexual behavior, binge/purge behaviors) measured short term [7]. Making behavioral changes in six behaviors some very resistant to change was considered surprising. But CDIs may have helped participants with behavioral skills at a critical moment. Interventions also affected psychological antecedents to change as predicted by theory. CDIs also allowed for tailoring of interventions to individuals. The authors caution that the results were based on a few studies considering that multiple behaviors were reviewed, the effects were immediate and may not be long-term, and computer-delivered interventions did not improve physical activity, weight loss or diabetes self-management. A more recent review of health promotion on Web 2.0 located only 34 pilot interventions (with the other articles being commentaries, reviews, or descriptive studies) and described the scarcity of research in this area recently [8]. The authors highlighted the need for more participatory and user-generated features to take advantage of Web 2.0 and more effectively enhance health promotion interventions. Clearly, more research is needed to evaluate the latest generation of computers and technology in health promotion. Future research should improve longterm efficacy and affect multiple behaviors. Some of this may be accomplished with continuing follow-up with the health promotion or longer-term efforts. Other areas to consider are making the interventions interesting and relevant to the particular populations.

In particular, although these reviews offer promise, more recent developments in technology might also further improve the findings. Virtual reality techniques with its realistic depiction of potential life experiences has great value in preparing people for real life encounters with behaviors they are trying to avoid or ways in which they wish to act. Adolescents and young adults might be especially interesting targets for health promotion and prevention using virtual reality due to their immersion with technology in play, school/work and lifestyle. Recommendations have been made to use this technology for health promotion with existing virtual reality sites online [9]. But collaborations between public health researchers and computer scientists may allow for greater specificity and creativity in the creation of a virtual reality health promotion intervention.

\subsection{Public Health Informatics}

Public health informatics is an interdisciplinary field combining components of computer science (mathematics, engineering, and information) as well as social science (e.g., decision-making) to public health issues [10]. The relatively new field of public health informatics served to create a system using portable handheld computers and electronic data systems to successfully implement global adult tobacco surveys in developing countries [11]. Specifically, using these techniques with more than 2,644 handheld computers, interviews in 302,800 households in 14 countries in 38 languages were conducted. The global hardware failure rate was $<1 \%$ and data loss was almost $0 \%$. This was an impressive endeavor given that in the past questionnaires had to be completed by paper and pencil; furthermore most of the countries lacked internet access making the task quite challenging. This is an example of a success story in the world of public health informatics especially because the effort involved developing countries.

Other researchers in public health informatics created a surveillance system using electronic medical records from primary physicians using algorithms to integrate primary care with public health departments to avoid extra work [12]. Although these systems have great potential, the authors realize patient care may be fragmented and not all conditions may appear in the primary physicians' electronic records plus the system needs to have enough cases to meet the definition. In addition, the authors acknowledge physicians may differ in workup, management and coding of the target conditions. These are among the promising breakthroughs in this area. Plans are underway to make medical records electronic. This is one area of great potential for the intersection between the disciplines of public health and computer science.

One major important opportunity that the CDC reported is for public health informatics to increase the adoption of electronic health records and health information exchange systems; moreover, as part of the American Recovery Reinvestment Act, hospitals have an incentive to modernize to electronic health records by 2014 [10]. As a result, many hospitals are making their patient records electronic, which can allow physicians at the same hospital caring for the same patient to access information about the patient's other medical care. In this way, physicians, at least at the same hospital, need not spend time contacting one another about a particular patient. Moreover, they will have access to medical tests such as a blood test making it possible for patients to avoid unnecessary repeated testing.

\subsection{Health Outcomes}

Health outcomes research focuses on outcomes or health care. Episode creation described as classifying medical events and related data to higher level concepts like diseases using a combinatorial optimization approach for constructing episodes demonstrated significant improvement over traditional healthcare approaches [13]. Older adults living in retirement communities wore an aceleramotor and GPS device for seven days and those who spent more than 30 minutes outdoors or in physical activity for depressive symptoms, fear of falling and selfreported functioning [14]. The authors demonstrated the feasibility of using this technology with older adults to further determine relationships and guidelines for physical activity with this group. Under different circumstances, such research with older adults might not be possible as it could be difficult for them to keep track of many variables. Even reporting on multiple behaviors can be hard in younger populations. Showing feasibility with this group 
was also promising as older adults can be averse to using new technology.

In a review article about using internet tools or mobile devices to improve asthma management, the field proved to be promising using small pilot studies with mixed results and remains in its infancy [15]. More work clearly needs to be done to determine whether the internet and/or mobile devices can help with asthma management or in managing other diseases, such as diabetes. These are a few of the preliminary examples of work combining health outcomes with computer science. Other combinations of ideas and further exploration of these examples need to be explored in future research.

\subsection{Connective Media}

The social media appear to be at the heart of the connection between public health and computer science. Computational models have explored how social media, such as Twitter, are changing public health; computer research filtered tweets and found health-related messages, yielding 1.6 million English health tweets from March 2009 to October 2010 [16]. The article described several studies that used Twitter data to span an information gap traditionally faced in public health when patient-directed programs depending on sustained effort by patients outside the clinic (e.g., smoking cessation, exercise). Specifically, 15,000 tweets were used to manage and share information about exercise and health promoting behavior with $10 \%$ of messages asking other users about exercise and many containing advertising. In another study smoking tweets were grouped into themes suggesting twitter is a promising tobacco data source for smoking behaviors and trends [17]. Mining this data from Twitter could impact many areas of public health, resulting in new public health research, practice and policy.

Blog posts from the US 2008 to 2009 season tracking the flu were found to significantly correlate with CDC reports making text and data mining of this social media source a novel flu surveillance resource and way to identify affected communities [18]. More than half (60\%) of state public health departments have adopted use of at least one social media site with Twitter predominant among these (87\%), Facebook accounting for 56\% and Youtube channel at 43\% [19]. Basically one message per day was posted and little interaction took place.

At this stage, the state public health departments were in early adoption, but the authors argue that the state public health departments need to be more interactive and encourage engagement to improve their engagement. Studying connective media has a promising start and further care needs to be taken to develop it further. Including computer science experts seemed to increase the best use of social media. This area should be expected to grow more as both individuals and institutions engage more and research more with social media. Moreover, social media sites will continue to multiply and develop as the internet changes further over time to become even more interactive and available to more people through not only computers but mobile devices as well. Finally, in this area it would also be advantageous to include social media experts in collaboration with public health researchers. This would help elucidate what factors of social media affect users and how these can be used to impact public health.

\section{Conclusions}

The material covered here is a taste of the early efforts of collaboration between the disciplines of public health and computer science, as well as examples of public health incorporating computer science and technology. Although some areas in public health are well on their way, many others are still in their beginnings with pilot studies or not enough evidence for effectiveness. The process must continue with more advances in technology and computer science to help public health. The internet and social media offer opportunities for new ways to engage the public. As noted earlier, e-health and m-health are important resources in the increasingly more technological world. Although plenty of public health organizations use the internet as an information source to the public, greater forms of interactivity would greatly enhance the public's participation.

Moreover, newer techniques in animation and virtual reality would be perfect ways for younger people (adolescents and young adults) to be involved in health promotion/prevention at a stage when it is critical to have them transition smoothly through the life changes of adolescence and increasing independence into young adulthood before problems develop (e.g., substance use, obesity, poor mental health) and to encourage healthy behaviors (e.g., physical activity, eating healthy foods like fruits and vegetables). Some of the preliminary work suggests that older adults were open to using newer technologies likes GPS and wearing aceleramotors. Robotics also offers possibilities as surgical techniques have incorporated robotics benefitting the public's health. Moreover, robotics can also assist older adults in tasks related to their daily life. In summary, many possibilities remain for collaboration between the disciplines of public health and computer science.

\section{Acknowledgement}

This material is based upon work supported by the National Science Foundation under Grant No. IIS1116186 awarded to the author.

\section{Conflicts}

The author has no competing interests.

\section{References}

[1] Fairfield,A.L., Rosner, D., Colgrave, J., Bayer, R. and Fried, L.P., "The exodus of public health what the history can tell us about the future," American Journal of Public Health,100(1). 54-63. Jan. 2010.

[2] CDC, "Ten great public health achievements, 1900-1999," Mortality and Morbidity Weekly Report, 48(12), 241-243.

[3] Nelson, N. "The majority of cancers are linked to the environment," BenchMarks, 4(3). 1-4. 2004.

[4] U.S. Department of Health and Human Services. The Health Consequences of Involuntary Exposure to Tobacco Smoke: A 
Report of the Surgeon General. Atlanta, Georgia: U.S. Department of Health and Human Services, Centers for Disease Control and Prevention, Coordinating Center for Health Promotion, National Center for Chronic Disease Prevention and Health Promotion, Office on Smoking and Health, 2006.

[5] Denning, P.J. “Computer science: the discipline,” Encyclopedia of Computer Science (A. Ralston and D. Hemmendinger, Eds), Wiley, New York, 2000.

[6] Perez-Pena, R. "Alliannce formed secretly to win deal for campus,” New York Times, A23, December 25, 2011.

[7] Portnoy, D.B., Scott-Sheldon, L.A.S., Johnson,B.T and Casey, M.T. "Computer-delivered interventions for health promotion and behavioral risk reduction: A meta-analysis of 75 randomized controlled trials, 1988 - 2007," Preventive Medicine. 47 (1 ), 3-16. July 2008.

[8] Chou, W.S., Prestin, A., Lyons,C, and Wen, K. "Web 2.0 for health promotion: reviewing the current evidence," American Journal of Public Health. 103(1). e9-e18. Jan 2013. doi:10.2105/AJPH. 2012.301071.

[9] Annang, L., Muilenburg, J.L.,and Strasser, S.M.” Virtual worlds: Taking health promotion to new levels," American Journal of Health Promotion, 24(5) 344-346. May/June 2010.

[10] Savel T.G. and Foldy S. "The role of public health informatics in enhancing public health surveillance," MMWR Surveillance Summary. 61 Suppl:20-24. July 272012.

[11] Pujari, S.,Palipudi, K,M., Morton, J., Levinsohn, J., Litavecz, S., and Green, M. "Electronic data collection and management system for global adult tobacco survey,” Online Journal of Public Health Informatics, http://ojphi.org 4( 2). 2012.

[12] Klompas, M., McVetta, J., Lazarus, R. Eggleston, E., Haney, G., Kruskal, B.A., Yih, W.K., Daly, P., Oppedisano, P., Beagan, B., Lee, M., Kirby, C., Heisey-Grove, D., DeMaria Jr, A. and Platt, R.
"Integrating clinical practice and public health surveillance using electronic medical record systems," American Journal of Preventive Medicine, 42 (6). Supplement 2. S154-S162. June 2012.

[13] Son, R., Taira, R.K, Kangarloo, H and Cardenas, A.F. “ContextSensitive correlation of implicitly related data: An episode creation methodology," IEEE Transactions On Information Technology In Biomedicine, 12(5). 549-560. Sept 2008.

[14] Kerr, J., Marshall, S., Godbole S., Neukam, S., Crist,K., Wasilenko,K., Golshan,S. and Buchner, D. "The relationship between outdoor activity and health in older adults using GPS," International Journal of Environmental Research and Public Health, 2012, 9(12), 4615-4625. December 10, 2012. doi:10.3390/ijerph9124615.

[15] Mosnaim, G.S., Powell, L.H., and Rathkopf, M. A. "Review of published studies using interactive internet tools or mobile devices to improve asthma knowledge or health outcomes," Pediatric Allergy, Immunology, And Pulmonology, 25(2), 2012.

[16] Dredze, M. "How social media will change public health," IEEE Intelligent Systems, 27(4), 81-84, 2012.

[17] Prier,K.W., Smith, M.S., Grand-Carrier, C. \& Hanson, C.L. "Identifying health-related topics on Twitter an exploration of tobacco-related tweets as a test topic," Social Computing, Behavioral Cultural Model and Prediction (J. Alerno, S.J. Young, D. Nau \& S. Chai, Eds). Springer-Verlag, Berlin, 2011.

[18] Corley, C.D., Cook, D.J., Mikler, A.R. \& Singh, K.P. "Text and structural data minining of influenza mentions in internet and social media,” International Journal of Environmental Research and Public Health, 7(2), 596-615, 2010.

[19] Thackeray, R., Neiger, B. L., Smith, A K. \&Van Wagenen, S.B. "Adoption and use of social media among public health departments,” BMC Public Health, 12, 2012. 